\title{
Selective Innervation of Transplanted Limb Muscles by Regenerating Motor Axons in the Axolotl
}

\author{
Donald J. Wigston \\ Department of Physiology, Emory University School of Medicine, Atlanta, Georgia 30322
}

\begin{abstract}
The segmental pattern of motor innervation of hindlimb muscles in the axolotl was studied before and after reinnervation. To ascertain the specificity of reinnervation, the four spinal nerves innervating the hindlimb were severed and allowed to regenerate. The segmental origin of axons reinnervating particular muscles was then determined by intracellular recording from muscle fibers. Muscles were reinnervated in a specific manner: From the outset, the axons reinnervating each muscle originated largely from segmentally appropriate spinal nerves in the proper proportions, suggesting that a reliable mechanism of selective synapse regeneration exists even in mature axolotls. To examine the selectivity of reinnervation, individual muscles were transplanted to novel positions within the limb and the specificity of their reinnervation determined. Even after being moved to new positions, muscles were reinnervated for the most part by axons of appropriate segmental origin. Therefore, cues must exist on or within limb muscles that regenerating motor axons recognize and use to discriminate hetween different muscles during synapse formation. These results suggest that one of the mechanisms that promote the reestablishment of correct connections during reinnervation of axolotl limbs may be the selective formation of synapses with appropriate target cells.
\end{abstract}

An important feature of the innervation of vertebrate muscles is its specificity: Defined pools of motor neurons innervate particular muscles exclusively. A great deal, if not all, of this specificity is thought to arise during embryonic development from the guidance of growing axons to the sites of their future targets (Lance-Jones and Landmesser, 1981; Landmesser, 1980; Tosney and Landmesser, 1985). The convincing evidence for directed axonal growth, however, has meant that additional mechanisms for generating specific connectivity are at risk of being ignored; i.e., once growing axons reach the periphery, they may express the ability to select between different classes of cells within a target-for example, fast and slow muscle fibers (Elizalde et al., 1983; McLennan, 1983) - and even between different muscles themselves (Sperry and Arora, 1965).

Common approaches to determining whether individual axons have a special affinity for their normal target have been (1) cutting a nerve supplying innervation to a group of different muscles and allowing it to regencratc (Bcrnstcin and Guth, 1961; Brushart and Mesulam, 1980; Sperry and Arora, 1965); (2) exchanging the nerves innervating 2 different sets of muscles (for example, flexors and extensors; Grimm, 1971; see also Sperry, 1941); or (3) encouraging native and foreign nerves to compete

\footnotetext{
Received Jan. 7, 1986; revised Mar. 28, 1986; accepted Apr. 1, 1986.

I am most grateful to D. Purves, J. Sanes, E. Frank, P. Kennedy, and A. English for helpful suggestions and encouragement, and to A. McLean for help in preparing the manuscript. This work was supported by grants from Emory University and the NIH.

Correspondence should be addressed to Donald J. Wigston at the above address.

Copyright (C) 1986 Society for Neuroscience $0270-6474 / 86 / 092757-07 \$ 02.00 / 0$
}

with one another to reinnervate a particular muscle (for example, see Bennett and Raftos, 1977; Dennis and Yip, 1978; Weiss and Hoag, 1946). The results of these kinds of experiments depend to a large extent on the species employed. In lower vertebrates, such as teleost fish and urodele amphibians, reinnervation apparently favors the restoration of appropriate connections (Dennis and Yip, 1978; Grimm, 1971; Holder et al., 1982; Sperry and Arora, 1965; Stephenson, 1979; see, however, Scherer, 1986; Wigston, 1980); on the other hand, the reinnervation of muscles in mammalian limbs appears to be relatively nonspecific (Brushart and Mesulam, 1980).

To test more directly for an ability of axons to choose between different potential targets (or for targets to select appropriate axons) during synapse formation itself, it is necessary to eliminate any requirement that axons first locate the right target before they can make appropriate connections. One way to achieve this and still retain sufficient flexibility for the expression of preferences between potential synaptic partners is to confront different muscles with a mixed population of axons, and then to determine which of the available axons make connections more effectively with particular muscles. For example, mammalian intercostal muscles transplanted to the neck from different thoracic segments can be distinguished by autonomic preganglionic axons in the cervical sympathetic trunk that arise from different segments of the spinal cord: Axons from upper thoracic spinal segments show a consistent preference for intercostal muscles from equivalent levels, whereas more caudally derived preganglionic axons reinnervate intercostal muscles transplanted from caudal thoracic segments more effectively (Wigston and Sanes, 1982, 1985).

I now report a series of experiments designed to determine whether limb muscles can be selectively reinnervated by motor neurons. The selective reinnervation of mammalian intercostal muscles according to their original rostrocaudal position suggested an approach to investigating the mechanisms underlying specific reinnervation of limb muscles since, like intercostals, different limb muscles normally receive motor innervation in approximate accordance with their position in the rostrocaudal axis. An important strategy during reinnervation, then, might be to match axons from particular spinal levels with muscles from appropriate rostrocaudal positions. Furthermore, it was important to determine whether motor axons, in addition to preganglionic axons, were capable of selective synapse formation, since it is already well established that sympathetic preganglionic axons can discriminate between different classes of superior cervical ganglion cells (Langley, 1895, 1897; Njå and Purves, 1977a, b), as well as distinguish sympathetic ganglia from different segmental levels (Purves et al., 1981). The results reported here on the reinnervation of transplanted muscles in axolotl hindlimbs show that the ability of motor axons to distinguish between different limb muscles is based, at least in part, on a muscle's original position in the rostrocaudal axis. A preliminary account has been reported elsewhere (Wigston, 1985). 


\section{Materials and Methods}

\section{Animals}

Male and female axolotls (Ambystoma mexicanum; wild-type) were obtained from a colony at Indiana University or from commercial suppliers. Animals used in this study ranged in length from 12 to $19 \mathrm{~cm}$, but were usually $16-18 \mathrm{~cm}$. They were kept in trays containing $20 \%$ modified Holtfreter's solution at $20 \pm 2^{\circ} \mathrm{C}$ and fed beef liver twice weekly.

\section{Surgery}

Prior to operating, animals were anesthetized in $0.1 \% \mathrm{MS} 222$ (tricaine methanesulfonate; Sigma) for $20 \mathrm{~min}$, laid in an operating dish, and covered with moist tissue. For limb denervation experiments, the nerves innervating the hindlimbs (spinal nerves $15-18$ ) were exposed by a dorsolateral approach and severed near their point of entry to the limb. Both left and right limbs were denervated in each animal. Skin incisions were closed with 6-0 sutures and animals returned to their containers.

For transplantation experiments, the anterior and posterior iliotibialis muscles (Francis, 1934) were exposed on the left side, removed together, and transferred to a dish of sterile Ringer's solution. The synonymous muscles were then removed from the right limb and sutured by their tendons into the left limb with 7-0 silk, as indicated in Figure 1; in this way the anterior iliotibialis from the right side occupied the position normally inhabited by the left posterior iliotibialis. The first pair of muscles from the left side was then implanted in the same way in the right limb. The skin was then sutured and the animals were returned to their containers. In each animal, then, 4 muscles were shifted about $1 \mathrm{~mm}$ in the rostrocaudal axis, 2 rostrally and 2 caudally. All 4 muscles were denervated by this procedure, since during removal the several small nerve branches that provide their innervation were severed close to their point of entry into the muscle. Since the innervation for both muscles arises from a common nerve trunk, which passes directly beneath them parallel to their long axis, it is reasonable to assume that during regeneration all axons have fairly equal access to both anterior and posterior muscles.

\section{Electrophysiological recording}

Normal and operated animals were pithed, eviscerated, and the lumbar region isolated by transection rostral to segment 14 and caudal to segment 19 . This hindlimb preparation was pinned in a dish of amphibian Ringer's of the following composition (mM): $\mathrm{NaCl}, 125 ; \mathrm{KCl}, 2 ; \mathrm{CaCl}_{2}$, 1.8; HEPES, 5; $\mathrm{NaOH}, 2$; dextrose, 11 (pH 7.2). Both hindlimbs were skinned and the ischium cut along the ventral midline. Spinal nerves 15-18 were severed at their exit from the vertebral column, freed to their entry to the hindlimb, and the leg removed by cutting the ilium as close as possible to the vertebral column. The isolated limb was trimmed of adherent body-wall musculature and transected below the knee; in some experiments the ventral flexor muscles were removed to reduce movement. The limb was placed on a thin layer of Sylgard (DowCorning) in a $35 \mathrm{~mm}$ petri dish, covered with Ringer's solution, and stabilized with pins through the ilium, ischium, and knee. Spinal nerves 15-18 were mounted in suction electrodes and the muscles checked visually for responses to nerve stimulation $(2-10 \mathrm{~V}$ pulses, $50 \mu \mathrm{sec}$ duration at $0.5 \mathrm{~Hz}$ ) with transillumination. The preparation was then perfused continuously with Ringer's containing $9 \mathrm{~mm} \mathrm{Mg^{2+ }}$ to reduce stimulus-evoked contractions. Muscle fibers in identified muscles were impaled with glass microelectrodes containing $3 \mathrm{M} \mathrm{KCl}$ (resistance, 20$40 \mathrm{M} \Omega$ ) and synaptic potentials were monitored on a storage oscilloscope. For each impalement, spinal nerves 15-18 were stimulated several times in turn, while the strength and polarity of the stimulus were varied to reveal multiple inputs (Redfern, 1970). Only fibers that yielded membrane potentials exceeding $-70 \mathrm{mV}$ after impalement were studied in detail; care was taken to sample fibers throughout the width and depth of each muscle. Generally a total of 10-12 satisfactory impalements was obtained from each muscle at about $100 \mu \mathrm{m}$ intervals.

\section{Analysis}

To analyze the fidelity of reinnervation in individual experiments, and to control for the variation between animals in the absolute level of origin of hindlimb innervation, a single value was assigned to each muscle, representing the average segmental origin of its innervation (Wigston and Sanes, 1982). A segmentally weighted average of all synaptic potentials recorded in a single muscle was calculated as follows:
If, in a muscle, 2 inputs were recorded from spinal nerve 16,8 from nerve 17 , and 2 from nerve 18 , that muscle would be assigned a value of $[(2 \times 16)+(8 \times 17)+(2 \times 18)] /(2+8+2)=204 / 12=17$. For each limb in which both the anterior and posterior muscles yielded 10 or more satisfactory impalements, the value obtained for the original anterior muscle was then subtracted from the value obtained for the original posterior muscle.

\section{Histology}

Either directly after dissection or following recording, muscles were fixed with $2 \%$ glutaraldehyde in $100 \mathrm{~mm} \mathrm{NaCl}$ buffered to $\mathrm{pH} 7.2$ with $5 \mathrm{~mm}$ HEPES. Muscles were then rinsed in buffer, dehydrated in an ethanol series, and embedded in paraffin. Sections $(10 \mu \mathrm{m})$ were cut on a sliding microtome and stained with toluidine blue for light microscopy.

\section{Results}

\section{Normal innervation of axolotl hindlimb muscles}

The dorsal muscles of the axolotl hindlimb are a convenient preparation for electrophysiological study. They are arranged in a simple rostrocaudal sequence and are easily accessible (Fig. 1). As a prelude to reinnervation experiments, the segmental innervation of 4 major proximal muscles was determined by recording intracellularly from myofibers in one or another of the muscles, while stimulating in turn the 4 spinal nerves that supply innervation to the hindlimb. All recordings were obtained in the presence of $9 \mathrm{mM} \mathrm{Mg}^{2+}$ to reduce muscle movement in response to nerve stimulation. For each impalement, the number and origin of all detectable synaptic inputs were noted. Since urodele muscle fibers generally have multiple endplate sites distributed along their length (Lehouelleur and Chatelain, 1974; Slack and Docherty, 1978), different axonal inputs could often be detected during a single impalement (Fig. $2 A$ ). On the basis of risetimes and differences in threshold, there were apparently several instances when more than 1 axon contacted a single synaptic site, either from the same (Fig. $2, B, D$ ) or a different (Fig. 2C) nerve. Recordings from a total of 880 fibers in 21 limbs (12 animals) yielded the results presented in Table 1 and Figure 3. The muscles differed systematically in the proportion of inputs that arose from axons in the 4 different spinal nerves (Fig. 3). For example, the most rostral of the 4 muscles, the pubo-ischio femoralis internus (PIFI; Francis, 1934), is innervated primarily by axons arising in spinal nerve 16 , with occasional contributions from axons in nerve 15 or 17 (Fig. 3a). The adjacent but more caudally located muscle, the anterior iliotibialis (ant ILT), received somewhat less innervation overall from nerves 15 and 16 , but substantially more from nerve 17 (Fig. 3b). Furthermore, posterior iliotibialis (post ILT) muscles differed markedly from both PIFI and ant ILT muscles in the source of their innervation; thus, virtually all the innervation of post ILT muscles arose from nerve 17 , with relatively little from nerve 16. A few post ILT muscles had some innervation from nerve 18 (Fig. $3 c$ ). Finally, the innervation of the iliofibularis (ILF), the most caudally located of the 4 muscles, received no contribution from axons in nerve 16 (Fig. $3 d$ ), although it was otherwise similar to post ILT. In summary, the rostrocaudal origin of each muscle's innervation was correlated with its rostrocaudal location within the limb.

\section{Reinnervation of normal muscles}

Reinnervation of denervated limb muscles was studied in 10 axolotls to determine the appropriateness of normal reinnervation. On both sides, spinal nerves 15-18 were severed at their point of entry to the limb. From 37 to $56 \mathrm{~d}$ later, the innervation of muscles in isolated limbs was examined as before with intracellular recording. The results for ant and post ILT are shown in Table 1 and Figure 4. The segmental origin of inputs to reinnervated ant ILT (Fig. 4c) was very similar to that of normal 


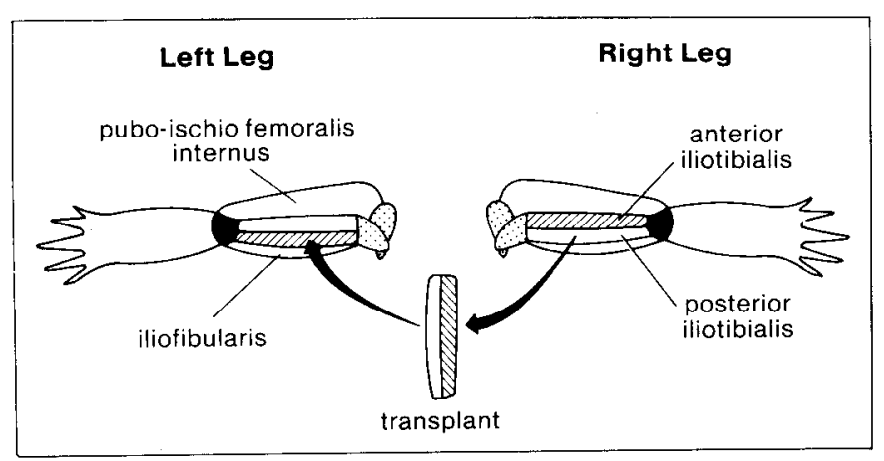

Figure 1. Diagram of the dorsal surface of left and right hindlimbs of the axolotl. The outlines of the 4 proximal muscles are shown in each limb: pubo-ischio femoralis internus (the most anterior muscle), anterior iliotibialis (cross-hatched), posterior iliotibialis and iliofibularis (the most posterior muscle). For transplantation experiments, ant and post ILT muscles were removed together as shown and implanted in the opposite limb in reversed configuration.

muscles (Fig. $4 a$; redrawn from Fig. 3 for comparison), except that a slightly higher proportion of inputs to reinnervated muscles came from nerve 17. The reinnervation pattern of posterior iliotibialis muscles (Fig. $4 d$ ) also closely resembled that of normal muscles (Fig. $4 b$ ), but with a somewhat greater contribution from nerve 18. This slight bias in favor of motor axons in more caudal spinal nerves was also apparent after removing muscles and reimplanting them in situ (Fig. 5, a, b); its cause, however, is unclear. More important, the normal distinction between the source of innervation to these 2 muscles was maintained after reinnervation: ant ILT muscles were again innervated by a more rostrally located set of motor neurons than were post ILT muscles.

\section{Reinnervation of transplanted hindlimb muscles}

To investigate the mechanisms responsible for the restoration of specific neuromuscular connections, a series of transplantation experiments were performed. These were intended to distinguish between 2 possibilities: first, that axons are specified or constrained to grow to a particular position within the limb and reinnervate whichever target they find there; and second, that synapse formation takes into account the identities of the pre- and postsynaptic cells so that axons reinnervate targets selectively.

Briefly, both iliotibialis muscles were removed together from one side and substituted for the same pair of muscles in the opposite limb, maintaining a normal dorsal/ventral orientation (Fig. 1). In this manner the ant and post ILT muscles were denervated and reversed in the rostrocaudal axis, so that, for each operated animal, 4 muscles were shifted in the rostrocaudal axis.

Several muscles were examined histologically at different times after transplantation to ascertain whether muscle fibers in the transplants survived intact, or whether a proportion of them degenerated and regencrated as in transplanted mammalian muscles (Hansen-Smith and Carlson, 1979; Wigston and Sanes, 1985). In contrast to findings in transplanted mammalian muscles, few degenerating fibers were observed, even at fairly short times after transplantation, although, as expected, myofibers showed somc atrophy. Thus, most fibers impaled during electrophysiological experiments had probably survived transplantation intact.

Intracellular recording 1-3 months after transplantation showed that the innervation of ant ILT muscles transplanted to the usual position of the post ILT (Fig. $5 c$ ) was consistently of more rostral origin than that of post ILT muscles that had been
A

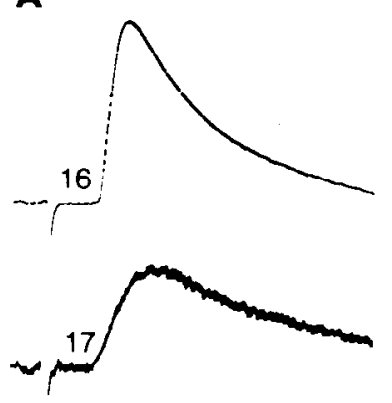

B

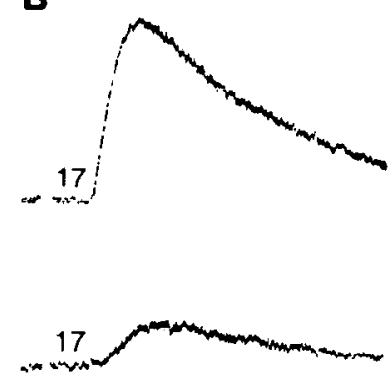

$\square$

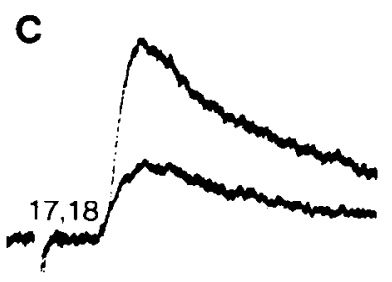

D

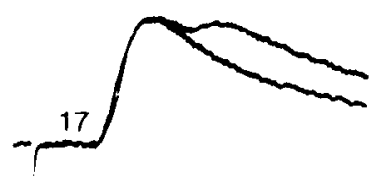

Figure 2. Examples of synaptic potentials recorded from axolotl muscle fibers in response to stimulation of individual spinal nerves. $A, \mathrm{~A}$ fiber that received inputs from 2 different axons, one from spinal nerve 16 and another from nerve 17 . The different risetimes of the resulting synaptic potentials suggests that these 2 inputs were at different endplate sites. $B$, A fiber innervated by 2 different axons, both from nerve 17, possibly at the same or 2 closely neighboring sites. $C$, A fiber receiving 2 inputs, a larger one from nerve 17 and a smaller one from nerve 18 , possibly at the same synaptic site. $D$, A fiber with 2 inputs from nerve 17 of considerably different latencies. In both $B$ and $D$, the upper trace was obtained at a higher stimulus intensity than the lower. $C$ and $D$, two traces are superimposed. Calibration bar: $A$, Upper trace, $10 \mathrm{mV}$ and $4 \mathrm{msec}$; Lower trace, $2 \mathrm{mV}$ and $4 \mathrm{msec} ; B, C$, all traces, $2 \mathrm{mV}$ and $4 \mathrm{msec} ; D, 4 \mathrm{mV}$ and $2 \mathrm{msec}$.

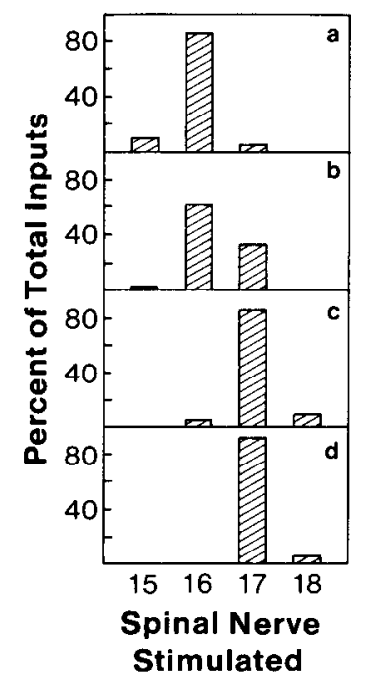

Figure 3. Segmental origin of normal hindlimb extensor muscle innervation. Percentages of all inputs from spinal nerves 15-18, recorded intracellularly. a, Pubo-ischio femoralis internus muscle fibers; $n=212$. $b$, Ant ILT muscle fibers, 218. $c$, Post ILT fibcrs, 238. $d$, Iliofibularis muscle fibers, 212. See Table 1 for details of ant and post ILT muscles. 


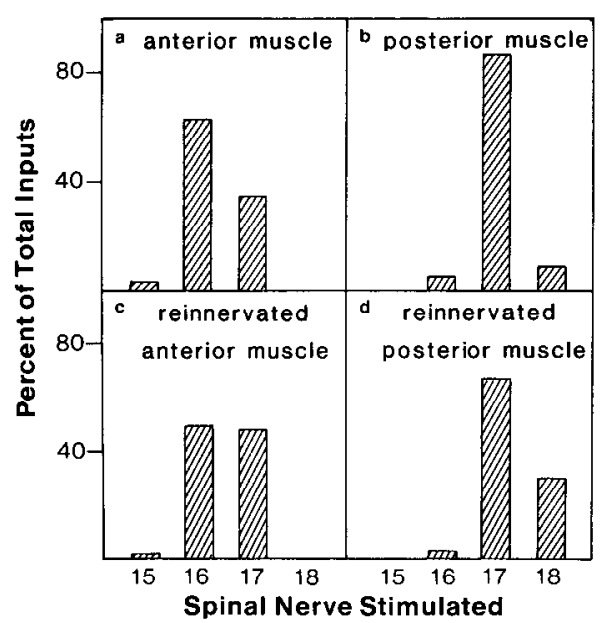

Figure 4. Segmental origin of inputs to reinnervated ant and post ILT muscles. $a, b$, Origin of inputs to normal muscles (redrawn from Fig. $3, b, c)$. $c$, Segmental origin of inputs recorded in ant ILT muscles $37-$ $56 \mathrm{~d}$ after cutting all nerves innervating the hindlimb. $d$, Origin of inputs to reinnervated post ILT muscles. Note similarity between $a$ and $c, b$ and $d$.

removed and reimplanted in their original position (Fig. $5 b$ ). Likewise, the innervation of transplanted post ILT (Fig. 5d) more closely resembled that of control post ILT muscles reimplanted in their own site (Fig. $5 b$ ), rather than reimplanted anterior muscles (Fig. 5a). Thus, both transplanted ant and post ILT muscles were reinnervated according to their initial, rather than their new, position (Table 2).

Another way of expressing the degree of selectivity in the reinnervation of transplanted ant and post ILT muscles is illustrated in Figure 6. The primary advantage of this method of analysis is that it cancels out the normal differences between animals in the spinal level of the motor neurons supplying their limb innervation. For example, some animals receive strong innervation from spinal nerve 15 , but none from 18 , while others receive none from 15 , but a considerable amount from 18 (Bennett and Raftos, 1977; Cass and Mark, 1975; see also Stephens and Holder, 1985). One way to control for these differences, which added considerable variability to the results used in rigures 3-5, is to reject animals that do not conform to certain criteria, such as having limbs that are innervated by nerve 18 (Bennett and Raftos, 1977). An alternative, less arbitrary, method, used in constructing Figure 6, is to examine the difference between the segmental origin of the innervation and pairs of iliotibialis muscles within individual limbs, so that the absolute spinal level of origin of their innervation is unimportant. Each point in Figure 6 represents a value calculated for a pair of

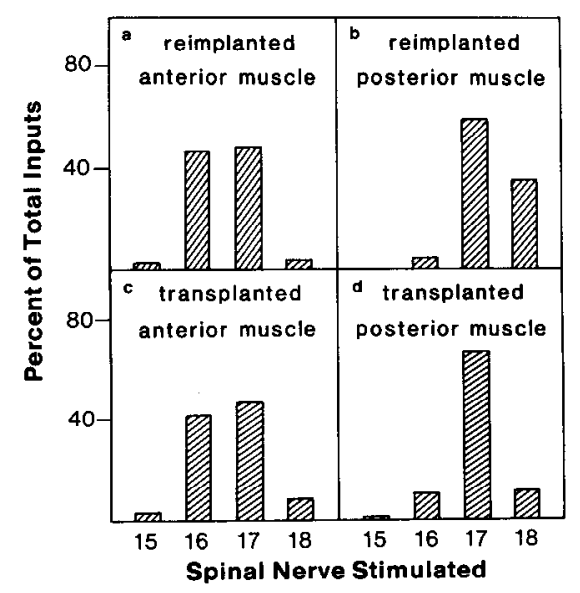

Figure 5. Segmental origin of regenerated synaptic inputs to transplanted ILT muscles. $a$, Control anterior and, $b$, control posterior ILT muscles removed and reimplanted in their original positions. $c$, Segmental innervation of ant ILT muscles transplanted to the normal position of the post ILT and reinnervated. $d$, Reinnervation pattern of post ILT muscles moved to the original site of the ant ILT. Compare $c$ with $a, d$ with $b$.

transplanted (dots) or reimplanted (triangles) ant and post ILT muscles that depends on the difference in the average segmental origin of their innervation (see Materials and Methods, and Fig. 6 legend for details). In this analysis, positive values represent pairs of ant/post ILT muscles in which the original ant ILT muscle received its overall innervation from a more rostrally located set of motor neurons than the original posterior muscle; in the case of transplanted muscles, this would be in spite of their rostrocaudal positions being reversed. Negative values, on the other hand, represent muscle pairs in which the original ant ILT muscle was reinnervated by axons of more caudal origin, on average, than those reinnervating the original posterior muscle. The vast majority $(43 / 52 ; 83 \%)$ of transplantation experiments yielded positive values by this analysis, indicating reestablishment of an appropriate difference in the overall segmental origin of axons innervating transplanted ant and post ILT muscles. In $83 \%$ of cases, then, the transplanted post ILT muscle was reinnervated selectively by a more caudal set of motor axons than the original anterior muscle, in spite of their positions being reversed.

Over the time course of the experiments, a potentially interesting point emerged: The fidelity of the reinnervation pattern appeared to improve somewhat with time after transplantation (see Fig. 6). For example, 17 of 22 muscle pairs (77\%) examined less than $40 \mathrm{~d}$ after transplantation produced positive values, and hence were reinnervated selectively according to their orig-

Table 1. Innervation and reinnervation of iliotibialis muscles

\begin{tabular}{lccccc} 
& \multicolumn{2}{l}{ Normal muscles } & & \multicolumn{2}{c}{ Reinnervated muscles } \\
\cline { 2 - 3 } & Anterior & Posterior & & Anterior & Posterior \\
\hline Number of muscles & 21 & 21 & 16 & 16 \\
Innervated fibers studied & 218 & 238 & & 184 & 179 \\
Total inputs & 275 & 300 & & 323 & 308 \\
Average segmental innervation $^{a}$ & $16.31 \pm 0.05$ & $17.05 \pm 0.05$ & & $16.45 \pm 0.07$ & $17.23 \pm 0.06$
\end{tabular}

${ }^{a}$ Mean \pm SE of values calculated separately for each muscle, as described in Materials and Methods: Lower values represent innervation from more rostral spinal nerves, and higher values more caudal spinal nerves. Muscles in which $<10$ innervated fibers were studied are not included. Significance of differences by Student's $t$ test: Normal (anteriorposterior), $p<0.0005$; reinnervated (anterior-posterior), $p<0.0005$; anterior (normal-reinnervated), $p<0.01$; posterior (normal-reinnervated), $p<0.025$. 
Table 2. Reinnervation of transplanted iliotibialis muscles

\begin{tabular}{|c|c|c|c|c|c|c|}
\hline & \multicolumn{2}{|c|}{ Reimplanted in situ } & \multicolumn{4}{|l|}{ Transplanted } \\
\hline & \multirow[b]{2}{*}{ Anterior } & \multirow[b]{2}{*}{ Posterior } & \multicolumn{2}{|l|}{ Short term ${ }^{a}$} & \multicolumn{2}{|l|}{ Long term ${ }^{b}$} \\
\hline & & & Anterior & Posterior & Anterior & Posterior \\
\hline Number of muscles & 23 & 23 & 40 & 38 & 39 & 39 \\
\hline Innervated fibers studied & 253 & 254 & 374 & 332 & 431 & 396 \\
\hline Total inputs & 409 & 438 & 631 & 499 & 789 & 678 \\
\hline Average segmental innervation ${ }^{c}$ & $\begin{array}{l}16.52 \pm 0.09 \\
(21)\end{array}$ & $\begin{array}{l}17.28 \pm 0.09 \\
(20)\end{array}$ & $\begin{array}{l}16.58 \pm 0.09 \\
(27)\end{array}$ & $\begin{array}{l}16.85 \pm 0.09 \\
(24)\end{array}$ & $\begin{array}{l}16.61 \pm 0.06 \\
(37)\end{array}$ & $\begin{array}{l}16.94 \pm 0.06 \\
(32)\end{array}$ \\
\hline
\end{tabular}

${ }^{a}$ Days after transplantation, $<40$.

- Days after transplantation, $\geq 40$.

${ }^{c}$ Mean \pm SE of values calculated separately for each muscle, as described in Materials and Methods: Lower values represent innervation from more rostral spinal nerves and higher values more caudal spinal nerves. Numbers of muscles included are in parentheses; those in which $<10$ innervated fibers were studied are excluded. Significance of differences by Student's $t$ test: Reimplanted (anterior-posterior), $p<0.0005$; short-term transplanted (anterior-posterior), $p<0.025$; long-term transplanted (anterior-posterior), $p<0.0005$; anterior (reimplanted-long-term transplanted), $p<0.2$; posterior (reimplanted-long-term transplanted), $p<0.0025 ;$ anterior (shortterm transplanted-long-term transplanted), $p<0.4$; posterior (short-term transplanted-long-term transplanted), $p<0.25$.

inal position. A slightly greater fraction $(14 / 17 ; 82 \%)$ of experiments conducted $40-60$ d following transplantation were positive. Thirteen muscle pairs were examined after $60 \mathrm{~d}$; all but 1 of these $(92 \%)$ were reinnervated appropriately. These findings suggest that the selectivity of initial reinnervation might be somewhat weaker than was inferred from muscles examined at later times, consistent with the possibility that some degree of sorting out or rearrangement of connectivity occurs after initial synapse formation (see also Brown and Booth, 1983). However, further work must be done to clarify this point-for example, studies at even earlier times after transplantation-since the apparent differences were not statistically significant by Student's $t$ test or by 1-way ANOVA.

\section{Discussion}

Hindlimb muscles in the axolotl were reinnervated by motor axons of similar segmental origins to those providing their original innervation. Moreover, reinnervation was appropriate even when muscles were shifted to a different position within the limb: Anterior iliotibialis muscles were reinnervated by axons originating from the usual spinal nerves, and in the proper proportions, even after they were placed in a position normally inhabited by the posterior iliotibialis. Likewise, post ILT muscles were reinnervated in an appropriate pattern after being moved to a more anterior position. This argues for the existence of a mechanism for reestablishing specific connections in the limb that takes into account some property of the target cells encountered by growing axons; clearly, regenerating axons do not just grow to a particular position and indiscriminately innervate any target cells they find there. The importance of these observations is that they demonstrate that different limb muscles can be distinguished from one another and be reinnervated selectively by regenerating motor axons.

Other studies of muscle reinnervation in urodele amphibians have suggested that motor neurons have a strong affinity for their original muscle and can suppress functional synapses formed by a foreign nerve (Bennett and Raftos, 1977; Cass and Mark, 1975; Dennis and Yip, 1978). However, in these experiments, the foreign nerve may have been at a competitive disadvantage for reasons unrelated to selectivity per se (Wigston, 1980). Therefore, it is not clear if the results of these earlier experiments adequately address the question of whether motor neurons have the ability to discriminate between different muscles. On the other hand, recent experiments have shown that the synapses formed in a muscle by a regenerating nerve have a lower quantal content than synapses that form when the muscle's original nerve is made to reinnervate it (Holder et al., 1982; see also Sayers and Tonge, 1982). One major difference between these latter experiments and those reported here is that, in my experiments, regenerating axons were given a choice: When presented with 2 muscles, motor axons seemed to selectively reinnervate their original muscle, at the expense of the foreign muscle. This result shows more clearly how selectivity in the process of synapse formation can translate to specificity in connectivity.

Another system in which selective reinnervation of different targets has been shown directly is the segmentally arranged collection of neurons within the mammalian sympathetic nervous system. If ganglia from different rostrocaudal levels of the sym-

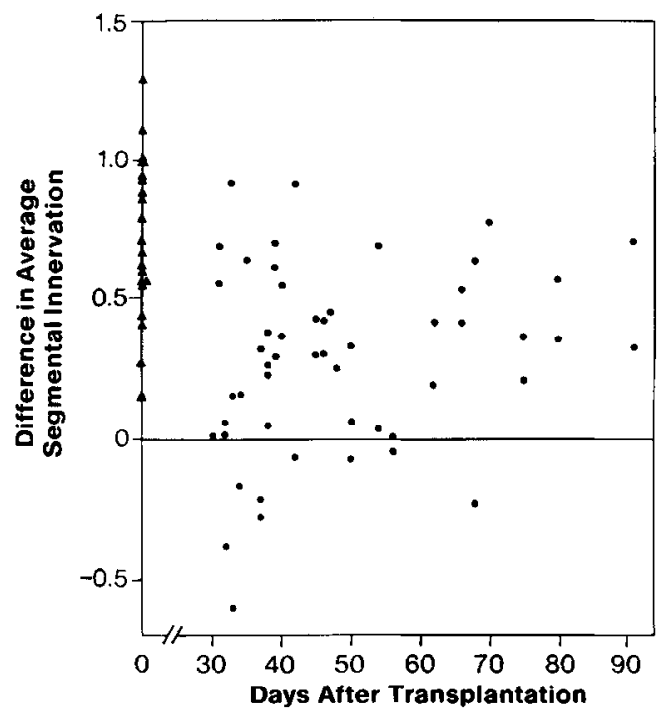

Figure 6. Difference in average segmental innervation of transplanted ant and post ILT muscles. As described in Materials and Methods, a segmentally weighted average was calculated for all synaptic inputs recorded intracellularly in the myofibers of each muscle. The value obtained for the ant ILT muscle was then subtracted from that obtained for the post ILT muscle in the same limb. The resulting index of innervation is shown for control muscles reimplanted in their normal positions (triangles at $t=0$ ) and experimental muscles transplanted to a new position in the rostrocaudal axis (dots). Positive values of this index represent pairs of muscles whose reinnervation pattern resembled that of normal muscles - that is, posterior muscles innervated by axons of more caudal origin, on average, than those innervating ant ILT muscles. For transplanted muscles, then, these positive values indicate those pairs of muscles reinnervated by segmentally appropriate sets of motoneurons in spite of their positions being reversed. Muscle pairs in which 1 or both muscles yielded satisfactory recordings from fewer than 10 muscle fibers are not included. 
pathetic ganglion chain are presented to the axons in the cervical sympathetic trunk by transplanting them to the position of the superior cervical ganglion, they are reinnervated selectively. For instance, preganglionic axons in the cervical trunk arising from lower thoracic segments of the spinal cord are more effective in reinnervating transplanted lumbar or thoracic ganglia than reimplanted superior cervical ganglia. Conversely, axons arising from upper thoracic segments reinnervate superior cervical ganglia more effectively than transplanted thoracic or lumbar ganglia (Purves et al., 1981). Ganglion cells from different segmental levels must differ, then, in some quality that selectively influences their reinnervation.

Selective synaptogenesis based on the origin of target cells in the rostrocaudal axis has also been demonstrated during reinnervation of mammalian intercostal muscles by sympathetic pregangliunic axons. Intercostal muscles transplanted to the neck from rostral thoracic segments are preferentially reinnervated by a subset of axons in the cervical trunk that arise from relatively rostral segments of the spinal cord, whereas intercostals transplanted to the same site from more caudal levels are innervated more effectively by axons from more caudal segments (Wigston and Sanes, 1982, 1985). Thus, segmentally arranged skeletal muscles apparently also differ somehow in a way that regenerating preganglionic axons can recognize.

The present results offer 2 significant advances from these earlier reports. First, motor axons, and not only autonomic preganglionic axons, which are well known to be capable of reinnervating subsets of sympathetic ganglion cells selectively (Langley, 1895, 1897; Njå and Purves, 1977b; Purves et al., 1981), can distinguish between different muscles and reinnervate them selectively. Second, limb muscles, which lack the clear-cut segmentation of intercostal muscles, can nevertheless be distinguished by regenerating axons (see also Holder et al., 1982; Sayers and Tonge, 1982). This recognition may also be related to a muscle's original position in the rostrocaudal axis, since this is the most clear-cut difference between the ant and post ILT muscles: Both contain virtually identical proportions of fiber types ( $\mathrm{N}$. Holder, personal communication) and probably have identical functions. Indeed, other studies have shown a correspondence between the rostrocaudal location of a motor neuron and the anterior-posterior position of its termination site (Brown and Booth, 1983; Landmesser, 1978; Laskowski and Sanes, in press; see also Fig. 3). The results reported here suggest that axons can discriminate between target cells possessing different recognition labels. Positionally related recognition labels may contribute to the generation of topographical rclationships.

Previous work has suggested that the reinnervation of mammalian limb muscles is disappointingly nonselective. In some of these studies (Bernstein and Guth, 1961; Brushart and Mesulam, 1980), mixed nerves were cut at a considerable distance from the muscles they supply and therefore regenerating axons were required first to grow to the right location before they could make appropriate connections. In others (Gerding et al., 1977; Weiss and Hoag, 1946), somewhat more direct tests of discrimination at the level of synapse formation were made, but still failed to reveal selectivity. Nevertheless, the discrimination of mammalian intercostal muscles from different segmental levels (Wigston and Sanes, 1982, 1985), and now of different limb muscles in axolotls is encouraging, since it suggests the existence of intrinsic recognition cues associated with different skeletal muscles that are somehow related to a muscle's position in the rostrocaudal axis. It is possible that similar recognition cues distinguish different mammalian limb muscles but that their presence has previously gone undetected.

While the idea of intrinsic recognition labels is an attractive one, other possibilities must be considered. For example, since the synaptic targets in the present experiments had been inner- vated previously, it is conceivable that they had been "branded" somehow by the axons that first innervated them. In this scheme, prior to initial innervation all muscles would be equivalent. After synaptogenesis, however, they might be modified by contact with particular axons and subsequently bear the mark of their initial association (see Schmidt, 1978). Since muscles that are forced to degenerate, but then regenerate, seem to lose their recognition cues (Wigston and Sanes, 1985), it might be possible to test this idea by reinnervating regenerated muscles prior to transplantation.

It is quite possible that the cues distinguishing the ant and post ILT muscles suggested by the present experiments might be related to the cues distinguishing different intercostal muscles, and sympathetic ganglia from different segments. Since each intercostal muscle is generated by myoblasts that migrate from a single somite (Chevallier, 1979) and is normally innervated by axons confined to about a single segment of the spinal cord (Dennis et al., 1981; Smith and Hollyday, 1983), intercostals could be labeled differentially and innervated according to the somite of their origin. Sympathetic ganglia, on the other hand, are derived from neural crest cells that arise primarily from the corresponding segment of the neural crest, with progressively smaller contributions from adjacent segments (Yip, 1983); their pattern of innervation mirrors this arrangement (Lichtman et al., 1980; Njå and Purves, 1977a). Like sympathetic ganglia, limb muscles also originate from more than 1 segment, so the segmental origins of different limb muscles can overlap (Chevallier, 1979). Nevertheless, individual muscles receive characteristic proportions of their myoblasts from particular somites in close correspondence with the source of their motor innervation (Beresford, 1983; Lance-Jones, 1985). The particular combination of myoblasts from different somites may impart a unique flavor to a muscle that labels it as an individual. Thus, a common thread linking the specific innervation and selective reinnervation of sympathetic ganglion cells, intercostal muscles, and limb muscles might be a general system of recognition labels related to their origin in the rostrocaudal axis.

\section{References}

Bennett, M. R., and J. Raftos (1977) The formation and regression of synapses during the re-innervation of axolotl striated muscles. J. Physiol. (Lond.) 265: 261-295.

Beresford, B. (1983) Brachial muscles in the chick embryo: the fate of individual somites. J. Embryol. Exp. Morphol. 77: 99-116.

Bernstein, J. J., and L. Guth (1961) Nonselectivity in establishment of neuromuscular connections following nerve regeneration in the rat. Exp. Neurol. 4: 262-275.

Brown, M. C., and C. M. Booth (1983) Postnatal development of the adult pattern of motor axon distribution in rat muscle. Nature 304: 741-742.

Brushart, T. M., and M.-M. Mesulam (1980) Alteration in connections between muscle and anterior horn motoneurons after peripheral nerve repair. Science 208: 603-605.

Cass, D. T., and R. F. Mark (1975) Re-innervation of axolotl limbs. I. Motor nerves. Proc. R. Soc. Lond. [Biol.] 190: 45-58.

Chevallier, A. (1979) Role of the somitic mesoderm in the development of the thorax in bird embryos. II. Origin of thoracic and appendicular musculature. J. Embryol. Exp. Morphol. 49: 73-88.

Dennis, M. J., and J. W. Yip (1978) Formation and elimination of foreign synapses on adult salamander muscle. J. Physiol. (Lond.) 274: 299-310.

Dennis, M. J., L. Ziskind-Conhaim, and A. J. Harris (1981) Development of neuromuscular junctions in rat embryos. Dev. Biol. 81 : 266-279.

Elizalde, A., M. Huerta, and E. Stefani (1983) Selective reinnervation of twitch and tonic muscle fibres of the frog. J. Physiol. (Lond.) 340: 513-524.

Francis, E. T. B. (1934) The Anatomy of the Salamander, pp. 370371, Clarendon Press, Oxford, U.K.

Gerding, R., N. Robbins, and J. Antosiak (1977) Efficiency of rein- 
nervation of neonatal muscle by original and foreign nerves. Dev. Biol. 61: 177-183.

Grimm, L. M. (1971) An evaluation of myotypic respecification in axolotls. J. Exp. Zool. 178; 479-496.

Hansen-Smith, F. M., and B. M. Carlson (1979) Cellular responses to free grafting of the extensor digitorum longus muscle in the rat. $\mathbf{J}$. Neurol. Sci. 41: 149-173.

Holder, N., J. Mills, and D. A. Tonge (1982) Selective reinnervation of skeletal muscle in the newt Triturus cristatus. J. Physiol. (Lond.) 326: 371-384.

Lance-Jones, C. (1985) The somitic origin of limb muscles in the chick embryo: A correlation with motor innervation. Soc. Neurosci. Abstr. 11: 975.

Lance-Jones, C., and L. Landmesser (1981) Pathway selection by chick lumbosacral motoncurons during normal development. Proc. R. Soc. Lond. [Biol.] 214: 1-18.

Landmesser, L. (1978) The distribution of motoneurones supplying chick hindlimb muscles. J. Physiol. (Lond.) 284: 371-389.

Landmesser, L. (1980) The generation of neuromuscular specificity. Annu. Rev. Neurosci. 3: 279-302.

Langley, J. N. (1895) Note on regeneration of pre-ganglionic fibres of the sympathetic. J. Physiol. (Lond.) 18: 280-284.

Langley, J. N. (1897) On the regeneration of pre-ganglionic and of post-ganglionic visceral nerve fibers. J. Physiol. (Lond.) 22: 215-230.

Laskowski, M. B., and J. R. Sanes (in press) Topographic mapping of motor pools onto skeletal muscles. J. Neurosci.

Lehouelleur, J., and A. Chatelain (1974) Analysis of electrical responses of newt skeletal muscle fibres in response to direct and indirect stimulation. J. Physiol. (Paris) 68: 615-632.

Lichtman, J. W., D. Purves, and J. W. Yip (1980) Innervation of sympathetic neurones in the guinea-pig thoracic chain. J. Physiol. (Lond.) 298: 285-299.

McLennan, I. S. (1983) The development of the pattern of innervation in chick hindlimb muscles: Evidence for specification of nerve-muscle connections. Dev. Biol. 97: 229-238.

Njå, A., and D. Purves (1977a) Specific innervation of guinea-pig superior cervical ganglion cells by preganglionic fibres arising from different levels of the spinal cord. J. Physiol. (Lond.) 264: 565-583.

$\mathrm{Njå,} \mathrm{A.,} \mathrm{and} \mathrm{D.} \mathrm{Purves} \mathrm{(1977b)} \mathrm{Reinnervation} \mathrm{of} \mathrm{guinea-pig} \mathrm{superior}$ cervical ganglion cells by preganglionic fibres arising from different levels of the spinal cord. J. Physiol. (Lond.) 272: 633-651.

Purves, D., W. Thompson, and J. W. Yip (1981) Reinnervation of ganglia transplanted to the neck from different levels of the guineapig sympathetic chain. J. Physiol. (Lond.) 313: 49-63.

Redfern, P. A. (1970) Neuromuscular transmission in new-born rats. J. Physiol. (Lond.) 209: 701-709.
Sayers, H., and D. A. Tonge (1982) Differences between foreign and original innervation of skeletal muscles in the frog. J. Physiol. (Lond.) 330: $57-68$.

Scherer, S. S. (1986) Reinnervation of the extraocular muscles in goldfish is non-selective. J. Neurosci. 6: 764-773.

Schmidt, J. T. (1978) Retinal fibers alter tectal positional markers during the expansion of the half-retinal projection in goldfish. J. Comp. Neurol. 177: 279-300.

Slack, J. R., and J. R. Docherty (1978) The pattern of innervation of a polyneural muscle: Axolotl iliotibialis. Cell Tissue Res. 186: 171180.

Smith, C. L., and M. Hollyday (1983) The development and postnatal organization of motor nuclei in the rat thoracic spinal cord. J. Comp. Neurol. 220: 16-28.

Sperry, R. W. (1941) The cffect of crossing nerves to antagonistic muscles in the hindlimb of the rat. J. Comp. Neurol. 75: 1-19.

Sperry, R. W., and H. C. Arora (1965) Selectivity in regeneration of the oculomotor nerve in the cichlid fish, Astronotus ocellatus. J. Embryol. Exp. Morphol, 14: 307-317.

Stephens, N., and N. Holder (1985) A horseradish peroxidase study of motoneuron pools of the forelimb and hindlimb musculature of the axolotl. Proc. R. Soc. Lond. [Biol.] 224: 325-339.

Stephenson, R. S. (1979) Axon reflexes in axolotl limbs: Evidence that branched motor axons reinnervate muscles selectively. Exp. Neurol. 64: $174-189$.

Tosney, K. W., and L. T. Landmesser (1985) Specificity of early motoneuron growth cone outgrowth in the chick embryo. J. Neurosci. 5: $2336-2344$.

Weiss, P., and A. Hoag (1946) Compctitivc rcinncrvation of rat muscles by their own and foreign nerves. J. Neurophysiol. 9: 413-418.

Wigston, D. J. (1980) Suppression of sprouted synapses in axolotl muscle by transplanted foreign nerves. J. Physiol. (Lond.) 307: 355366.

Wigston, D. J. (1985) Selective reinnervation of axolotl limb muscles. Soc. Neurosci. Abstr. 11: 976.

Wigston, D. J., and J. R. Sanes (1982) Selective reinnervation of adult mammalian muscle by axons from different segmental levels. Nature 299: 464-467.

Wigston, D. J., and J. R. Sanes (1985) Selective reinnervation of intercostal muscles transplanted from different segmental levels to a common site. J. Neurosci. 5: 1208-1221.

Yip, J. W. (1983) Formation of paravertebral sympathetic chain ganglia in chick embryos. Soc. Ncurosci. Abstr. 9:937. 